\title{
ReALLEN: structural variation discovery in cancer genome by sensitive analysis of single-end reads.
}

\author{
Ryo Kanno ${ }^{1,2}$, Daisuke Tanaka ${ }^{1}$, Hideaki Nanamiya ${ }^{1}$, Takao Isogai ${ }^{1}$ \\ ${ }^{1}$ Translational Research Center, Fukushima Medical University, 1, Hikarigaoka, Fukushima city, \\ Fukushima, 960-1295, Japan \\ 2 Okinawa Institute of Science and Technology, 1919-1, Tancha, Onna-son, Okinawa, 904-0495, \\ Japan
}

To whom correspondence should be addressed: Ryo Kanno, Ph. D., Okinawa Institute of Science and Technology, 1919-1, Tancha, Onna-son, Okinawa, 904-0495, Japan

Telephone: +81-98-966-8514; E-mail: kanno.ryo@gmail.com

\begin{abstract}
Background: The structural abnormalities in chromosomes are important issues in cancer genomics. Next generation sequencing technologies have big potentials to detect the structural variations precisely and comprehensively. Nevertheless, it is still difficult problem to detect large structural variations from short read sequence data. Major efforts have been achieved with paired-end reads, since discordant pairs directly reflect the existence of large rearrangement. Furthermore, approaches to detect structural variations from single-end reads are still worthwhile challenge because they allow wide choices of sequencing platforms.
\end{abstract}

Result: We present ReALLEN, a series of tools to detect genomic rearrangement with base-pair resolution from single-end reads provided by next generation sequencing. We examined the performance of ReALLEN using simulated dataset and real dataset sequenced by Ion
Torrent systems. In most cases on the simulated dataset, ReALLEN showed nearly 100\% precision and better sensitivity than other major tools. Notably, ReALLEN showed stable scores even if it was on some unfavorable conditions, for example, low coverage or small variant size. On the real dataset sequenced by Ion Torrent systems, ReALLEN accurately found an insertional translocation that was crucial for the diagnosis of chronic myeloid leukemia.

Conclusion: ReALLEN is useful to researchers in finding genomic rearrangements. It will contribute to discovery of cancer-specific fusion proteins, precise diagnosis of known types of cancers, and understanding of genetic diseases caused by abnormal chromosomes.

Keyword: whole genome sequencing, next generation sequencing, rearrangement, structural variation, software, split read, cancer 
Structural variation discovery in cancer genome

\section{Background}

Normal cells could postnatally turn into cancer cells by acquisition of somatic mutations in their genome [1]. The mutations that affect large sequence alteration (typically $>50 \mathrm{bp}$ ) are called structural variations (SVs), and are distinguished from single nucleotide variations (SNVs) [2, 3]. The somatic rearrangement of chromosomes that produces deleterious fusion protein, for example, BCR-ABL, PML-RAR $\alpha$, or EML4-ALK, is known to promote carcinogenesis [4]. Although some fusion proteins have been identified by traditional approaches [3, 4, 5], systematic information for various types of cancer diagnosis is still insufficient. The comprehensive discovery of $\mathrm{SVs}$ in cancer genome will make it possible to find novel fusion proteins, and will contribute to the targeted cancer therapies. Next generation sequencing (NGS) technologies enable us to get vast amount of genetic information with lower cost, and more and more whole genome sequencing (WGS) data are accumulating $[6,7]$. Nevertheless, there is no gold standard method for the discovery of SVs from short read data.

Four distinct approaches have been reported to detect SV signatures from NGS data: Read-pair (RP), Read-count (RC), Split-read (SR) and de novo assemble (AS) [2, 8]. BreakDancer, DELLY, LUMPY, and PRISM are able to detect SVs from paired-end (PE) reads with RP or complexed methods of RP and SR [9, 10, 11, 12]. HiSeq systems (Illumina) produce PE data [7], and properly cooperate with these tools. On the other hand, other systems that produce only single-end (SE) data, including the Ion Proton (Thermo Fisher Scientific), cannot utilize them effectively. SR is generally lower at the sensitivity than RP, because SR can find only breakpoint (BP) that crosses just over a read, whereas RP can find BP in large gap between paired reads. However, it is an advantage that SR yields base-pair resolution. Besides, SR becomes a more powerful approach as the readable length by NGS gets longer. Socrates, CREST, Gustaf, and SPLITREAD detect SVs from SE data with SR-based methods [13, 14, 15, 16, 17].

We present new software, ReALLEN (Rearrangement Analysis by Long-Leap Estimation on NGS data), for the discovery of SVs with base-pair resolution from SE data. Notably, it has three features: (i) ReALLEN is specifically designed for cancer genome data. The content of reads with somatic mutations is usually very little in cancer sample, because most cancer tissues heterogeneously include both normal DNA and cancer DNA. Thus, high sensitivity is necessary to detect the weak SV signatures in cancer samples. ReALLEN improves the sensitivity by integrating two different SR-based algorisms, soft-clip split [18] and fixed-length split. (ii) ReALLEN is able to find both small SVs (deletions, inversions, and tandem duplications range from 25 bp to 400 bp) and large SVs (inter-chromosomal and intra-chromosomal translocations; deletions, inversions, and tandem duplications more than $400 \mathrm{bp}$ ). The existing RP-based methods have more or less problems to find the small SVs, although such 
Structural variation discovery in cancer genome

SVs are important in coding regions. (iii) ReALLEN is optimized for SE data. Because ReALLEN has been developed for Ion Torrent platforms at the beginning, all parameters has been designed to provide sufficient result without PE data. In spite of the primal purpose, ReALLEN will work well on various sequencing platforms, considering that it showed sufficient scores on simulated dataset as well as on real dataset sequenced by Ion Torrent systems.

\section{Implementation}

The main algorithm of ReALLEN was implemented in Ruby. Startup scripts for easy-run were implemented in Bash script. ReALLEN requires aligned sequence data in SAM or BAM format as input. ReALLEN outputs the position of SVs in CSV format with annotations or in simple BLAST-like format [19]. ReALLEN is designed to work in cooperation with any mapping software although we recommend BWA-mem [20, 21] and TMAP (Thermo Fisher Scientific). References and annotations for hg19 (UCSC human genome 19) are included in build-in package. We show the workflow of ReALLEN in Figure 1. The steps are as follows:

\section{Mapping filter.}

At the first step, ReALLEN removes well-mapped reads to achieve fast calculation because these reads rarely have BPs. ReALLEN counts mismatch alignments (insertion, deletion, and soft-clip) of each read, and discards reads in which the count does not exceed threshold. Applying larger threshold values at this step increases the entire speed of the calculation while it slightly decreases the sensitivity.

2. Soft-clip split and realignment.

If a read has long soft-clipped sequence, the read is separated into the soft-clipped end and the opposite end. Both ends of the read are realigned by BWA-mem.

3. Fixed-length split and realignment. If a read does not have enough length of soft-clipped sequence, the read is separated into two ends with fixed length. The reads with incorrect alignment are rescued at this step. Both ends of the read are also realigned by BWA-mem.

4. Mapping uniqueness filter.

If a read is not uniquely mapped to the reference by the realignment, the read is removed. The uniqueness is evaluated by the difference of the scores between primary alignment and secondary alignment. This step is significantly effective to reduce false positive, especially when we target the sequence which has repetitive regions. Low quality mappings are also removed at this step.

\section{Pairing test}

The filtered reads are paired with the opposite ends of the original reads. The pairing test is performed to find discordant pairs. If two reads of a pair are located on different chromosomes or inconsistent orientations, the pair is discordant. Besides, if the gap ( $\left.\mathrm{L}_{\mathrm{gap}}\right)$ is larger than the given size (the minimum SV size), the pair is discordant. $\mathrm{L}_{\text {gap }}$ is defines as

$$
\mathrm{L}_{\text {gap }}=\left|\mathrm{L}-\mathrm{L}^{\prime}\right|
$$


Structural variation discovery in cancer genome

where $\mathrm{L}$ is the length of the original read and $L^{\prime}$ is the distance between the realigned terminals of the read pair. The discordant pairs are the candidates of the BPs.

6. Search accurate positions of breakpoints.

Since the accurate positions of BPs cannot be identified by fixed-length split algorithm, ReALLEN calculates them by split mapping algorithm $[11,16]$. The original reads are mapped to the reference around the putative BPs in a way that allows a jump from one region to another region.

\section{Clustering and counting filter.}

The discordant pairs are classified by modified $\mathrm{k}$-means clustering. The radius ( $\mathrm{r}$ ) of clusters is given instead of number of clusters. A discordant pair, defined as an element, has six values: the chromosome $\left(\mathrm{c}_{1}\right)$, the position $\left(\mathrm{p}_{1}\right)$, and the orientation $\left(\mathrm{d}_{1}\right)$ of one end (left-end) and the chromosome $\left(\mathrm{c}_{2}\right)$, the position $\left(\mathrm{p}_{2}\right)$, and the orientation $\left(d_{2}\right)$ of the opposite end (right-end). The order of chromosomes is uniquely defined (e.g. chromosome $1<$ chromosome 2). The left-end and the right-end are swapped if $c_{2}$ is smaller than $c_{1}$ or $p_{2}$ is smaller than $p_{1}$. Each element is assigned to the cluster that firstly satisfies the following conditions:

$$
\begin{aligned}
\max \left(\left|\mathrm{p}_{1}-\widetilde{\mathrm{p}_{1}}\right|,\left|\mathrm{p}_{2}-\widetilde{\mathrm{p}_{2}}\right|\right) \leq r \\
\mathrm{c}_{1}=\widetilde{\mathrm{c}_{1}} \\
\mathrm{c}_{2}=\widetilde{\mathrm{c}_{2}} \\
\mathrm{~d}_{1}=\widetilde{\mathrm{d}_{1}} \\
\mathrm{~d}_{2}=\widetilde{\mathrm{d}_{2}}
\end{aligned}
$$

where $\widetilde{c_{1}}, \widetilde{c_{2}}, \widetilde{p_{1}}, \widetilde{p_{2}}, \widetilde{d_{1}}$, and $\widetilde{d_{2}}$ are median values for $\mathrm{c}_{1}, \mathrm{c}_{2}, \mathrm{p}_{1}, \mathrm{p}_{2}, \mathrm{~d}_{1}$, and $\mathrm{d}_{2}$ of all elements in that cluster, respectively. If no cluster satisfies the conditions, the element is assigned to a new cluster. These processes are repeated until all elements are classified. After the classification, the cluster that have less than $\mathrm{N}$ elements are removed ( $\mathrm{N}$ is a threshold value). When we suppose that error read is aligned to a random position, the possibility to align $\mathrm{N}$ error reads to an identical position is $(1 / s)^{N-1}$, where $s$ is size of reference. Therefore, taking more than 1 for $\mathrm{N}$, the false positive rate would be suppressed even if the size of reference increased.

\section{Local detection.}

This optional step is effective to find specific fusion proteins. Ideally, a rearrangement which produces abnormal protein is supposed to occur within exons or around exons. Besides, the limited number of genes can be targeted for cancer analysis [22]. ReALLEN keeps only BPs that connect the selected regions when turning on this option, and enables us to focus on the important genes. We will decrease the false positive rate significantly if we have well-chosen list of target genes.

9. Annotation and secondary filtering. We prepare gene names, exon regions, and cytobands as annotations for hg19. All annotations can be attached to the output and viewed in CSV table. Simple estimation of SV type and secondary filtering can be applied at this step.

\section{Results and Discussion}

\section{Preparation of simulated dataset}

In order to reduce $\mathrm{CPU}$ time for the 
Structural variation discovery in cancer genome

calculation, we used short partial sequences of hg19 for simulated dataset. The regions were carefully chosen not to bring sequencing bias after a preliminary test (data not shown). The artificial sequences (test sequence \#1) with inter-chromosomal and intra-chromosomal translocations were generated as follows: we randomly chose 20 positions on the partial sequences of hg19 (60,000,000-61,000,000 on chromosome 16 and 22,000,000-23,000,000 on chromosome 21), divided them into pieces at the positions, and randomly connected the pieces to reconstruct two rearranged sequences. 8 patterns of the sequences were individually created in this way.

The artificial sequences (test sequence \#2) with specific SV types were generated as follows: we inserted SVs of each type and each size category at 10 positions that we randomly chose on the partial sequence of hg19 $(60,000,000-61,000,000$ on chromosome 16$)$. The SV types were deletion, inversion, and tandem duplication. The size categories were 25-49 bp, 50-99 bp, 100-199 bp, 200-399 bp, 400-799 bp, and 800-1599 bp. 8 patterns of the sequences were individually created in this way.

Dataset \#1 and dataset \#2 were generated from test sequence \#1 and test sequence \#2, respectively. The artificial sequences were mixed with the corresponding normal sequences at a ratio of $50 \%$, which imitated autosomal heterozygous germline event. By the simulation using wgsim [23], the read data were generated at each condition (coverage, read type, and read length). The coverages were
$5 \times, 10 \times, 20 \times$, and $50 \times$. The read types and the read lengths were 100 bp PE, 200 bp SE, 300 bp SE, and 400 bp SE. The insert size of PE was $300 \mathrm{bp}($ sigma $=50)$. The generated reads were mapped to hg19 by BWA-mem with default parameters.

Dataset \#3 was generated from test sequence \#1. The parameters of wgsim were the same as dataset \#1. The reads in dataset \#3 were aligned by BWA-mem with different parameters. Match scores ('-A' option on BWA-mem) were 1 and 3 for Alignment \#1 and Alignment \#2, respectively. The larger match score for Alignment \#2 allowed incorrect mapping with less soft-clipping.

\section{Comparison with other SR-based tools}

We examined the performance of ReALLEN and other SR-based tools: CREST [14], Socrates [13], and Lumpy (only SR mode) [10]. SVs called by these tools on the simulated dataset (dataset \#1, \#2, and \#3) were classified into true positive (TP), which agreed with the correct BP, and remaining false positive (FP). A tolerance of $500 \mathrm{bp}$ was allowed even if the called position by each tool was shifted from the correct BP. Redundant calls for a single event were counted as one TP. The BPs not identified were counted as false negative (FN). Precision, Recall, and F-value were defined as follows:

$$
\begin{gathered}
\text { Precision }=\mathrm{TP} /(\mathrm{TP}+\mathrm{FP}) \\
\text { Recall }=\mathrm{TP} /(\mathrm{TP}+\mathrm{FN}) \\
\mathrm{F}-\text { value }=\frac{2 \cdot \text { Recall } \cdot \text { Precision }}{\text { Recall }+ \text { Precision }}
\end{gathered}
$$

Mean shift was average distance between the 
Structural variation discovery in cancer genome

correct BP and the called position. Precisions, Recalls, F-values, and Mean shifts on the simulated dataset were calculated (Table S1, Table S2, and Table S3).

The performance of ReALLEN on the low coverage conditions was compared with those of the other SR-based tools. Figure $2 \mathrm{~A}$ shows the performance to find large translocations on dataset \#1. The entire graphs indicate a trend that the higher coverage and the longer read length bring better performance. On the coverage of $50 \times$, all tools showed F-values close to 1.0 with $200 \mathrm{bp}, 300 \mathrm{bp}$, and $400 \mathrm{bp}$ reads (Fig. 2A). This implies that there are no significant difference among these tools on the high coverage conditions. On the other hand, ReALLEN showed the highest F-values on the coverages of $5 \times$ and $10 \times$ with any of the read lengths. The difference was obvious on dataset $\# 2$. Figure $2 \mathrm{~B}$ shows the performance to find SVs of the specific types and the size categories on dataset $\# 2$ on the coverage of $10 \times$ with 300 bp reads. ReALLEN showed the highest F-values for the small deletions and the small tandem duplications, without significant reduction compared to large SVs. The F-values of CREST and Lumpy (SR) were clearly decreased for the small deletions $(<100 \mathrm{bp})$ and the small tandem duplications $(<200 \mathrm{bp})$. The effect was weaker for Socrates than CREST or Lumpy (SR) although it was not a negligible quantity. For the small inversions, all of these tools showed lower F-values. Overall, these SR-based tools called highly precise positions of BPs with mean shifts less than $2 \mathrm{bp}$ (Table S1 and Table S2). Precisions by these tools were nearly 1.0 in almost every cases (Table S1 and Table S2). As a result of comparison with each tool, the performance of ReALLEN on the low coverage conditions was better than those of the other SR-based tools.

The effect of misalignment was examined in Figure 2C. Socrates showed the second best score to find translocations, deletions, and tandem duplications on dataset $\# 1$ and dataset \#2. One of the difference between ReALLEN and Socrates is the usage of fixed-length split. This approach will affect the dependency on the first alignment. It is indicated that the performance of simple soft-clip split algorithm is affected by the result of aligner [13]. Figure 2C shows the performance on two different mappings with the same read data (dataset \#3). By increasing the match score, the mapping was more tolerant of mismatches and indels. This imitated, or emphasized, the situation where aligner needed to allow discordant bases in alignment due to sequencing errors. When we increased the match score for the mapping, the F-values of Socrates clearly decreased, while the F-values of ReALLEN were nearly unchanged (Alignment $\# 2$ in Fig. 2C). Additionally, we tested dataset with high error rate (Fig. S1A and Table S4). The reduction of the performance of ReALLEN was smaller than that of Socrates on the dataset with $5 \%$ error rate. We examined the effect of the fixed-length split step, by comparing the results with or without this step (Fig. S1B and Table S3). We found that the performance for dataset\#3 without the fixed-length split step was slightly 
Structural variation discovery in cancer genome

lower than that with this step. Although it is difficult to evaluate which process actually contributed to the improvement of the performance, the fixed-length split step certainly affected the rescue of the inaccurate mappings. These results suggest that ReALLEN, which uses the combination of two algorithms, is rather independent on misalignment and resistant to sequencing errors.

\section{Comparison with RP-based tools}

- The average read length is important for the performance, and it is dependent on the sequencing platforms. Generally, single-end platforms are able to sequence longer reads than pair-end platforms [7]. Therefore, we compared the scores of long SE reads (300 bp) by ReALLEN with the scores of short PE reads (100 bp) by Delly [9] and Lumpy (SR+RP mode) [10] on the simulated dataset (Table S1 and Table S2). When we compared the F-values for large translocations on dataset \#1, there was no significant difference on the coverages of $20 \times$ and $50 \times$, and ReALLEN showed a little better score on the coverage of $5 \times$ than the other tools (Fig. 3A). The result suggests that ReALLEN is comparable in performance to the RP-based tools. Figure 3B is focused on the specific types and the size categories on the coverage of $10 \times$. All tools correctly called SVs more than 400 bp. However, Delly and Lumpy were much worse at calling the deletions ranged from $25 \mathrm{bp}$ to $200 \mathrm{bp}$ than ReALLEN. Delly and Lumpy rarely called the tandem duplications ranged from 25 bp to $400 \mathrm{bp}$.
According to the low performance, RP-based methods with short PE data is inadequate for the discovery of small SVs. SR-based methods with long SE data have advantage in this respect. As a result of comparison with each tool, ReALLEN could sensitively find small SVs in contrast to the RP-based tools.

\section{Preparation of real dataset}

The real dataset was obtained as follows: The fragment libraries of the CML sample (Supplier: ILSbio; Barcode: ILS27378; Sample ID: BM; Format: Frozen; Sample Type: Tumor; Organ Type: Bone Marrow; Species: Homo sapiens; Sex: F; Age: 50; Clinical Diagnosis: Leukemia; Pathological Diagnosis: Suggestive of Chronic Myeloid Leukemia; Description of Diagnosis: CML; Cancer Cell \%: n/a; Consolidated STAGE: n/a; Grade: n/a) were constructed with Ion Xpress Plus Fragment Library kit (Thermo Fisher Scientific). Emulsion PCR and chip loading were performed with the Ion Chef system (Thermo Fisher Scientific). The five runs of sequencing (two runs with the 540 chip and three runs with the 530 chip) were performed by the Ion S5XL (Thermo Fisher Scientific). The Ion S5XL with the 530 chip produced long (about $300 \mathrm{bp}$ ) SE data, and that with the 540 chip produced relatively short (about 200 bp) SE data (Table S5). The sequence data were processed by the standard Ion Torrent software including TMAP. Because Socrates and Lumpy did not work on the direct output of TMAP, the reads for these tools were reprocessed by BWA-mem instead of TMAP. All read data of the five runs were 
Structural variation discovery in cancer genome

merged for the test (Table S5).

\section{Performance test with real dataset}

We examined the performance of ReALLEN using the real dataset of the CML sample sequenced by Ion Torrent systems. The SVs on the dataset were detected by ReALLEN, Socrates, CREST, and Lumpy (only SR mode). According to the output of these tools, we found inter-chromosomal translocations, $t(9 ; 22)(q 34.1 ; q 11.2)$. All of the tools called the translocation from 23,632,280 in chromosome 22 to $133,708,253$ in chromosome 9 (Fig. 4A). The translocation corresponded to well-known rearrangement which produced BCR-ABL1 fusion protein found in CML patients $[2,24$, 25]. In addition, some of the tools called the translocation from 136,052,205 in chromosome 9 to $23,632,280$ in chromosome 22 , and the translocation from 133,708,255 in chromosome 9 to $136,052,210$ in chromosome 9 . These three translocations indicated that a fragment of 2.3 million base pair on chromosome 9 was inserted into chromosome 22 (Fig. 4B). Only ReALLEN called all of the three BPs, while other tools called the deficient parts of them (Fig. 4C and Fig. S2). Even if the dataset did not have plenty of read data (the coverage was 14.8×; Fig. S3), ReALLEN showed excellent sensitivity to find the three BPs necessary for identifying the large insertional translocation in the CML sample.

We investigated the detail of individual SVs called by these tools. ReALLEN called total $18,168 \mathrm{SVs}$ in the sample (Table S6). After secondary filtering, it kept 6,019 SVs. Socrates called 2,978 SVs (paired results). The total number of calls by ReALLEN, Socrates, and their overlap are summarized in Figure 5A and Figure 5B. Most SVs uniquely detected by ReALLEN were the deletions less than $400 \mathrm{bp}$ and the tandem duplications less than 100 bp (Fig. 5B). The result implies two things: Firstly, these small deletions and tandem duplications are major population of SVs in the actual cancer sample. Secondary, ReALLEN has potential to find SVs of these sizes with superiority to other existing tools. When we randomly sampled SVs called by ReALLEN and Socrates, their calls were judged substantially correct by manual validation (Table S7 and Fig. S4). Therefore, the large number of calls by ReALLEN reflects not low precision but high sensitivity.

\section{Performance test with public dataset}

In addition to our dataset, we examined the performance of ReALLEN using public dataset. We downloaded the benchmark SV calls [26, 27] for one individual in 1000 genome project (NA12878) [28, 29] from National Institute of Standards and Technology (NIST). The read data sequenced by the Ion Proton (ID: SRR1238539; Number of Bases: 32.9 G; Coverage: 11×; Mean Read Length: 176.8 bp; Read Type: single-end) were downloaded from Sequence Read Archive (SRA) [30] of National Center of Biotechnology Information (NCBI). We detected SVs by ReALLEN and Socrates, and extracted the deletions more than $50 \mathrm{bp}$ for the comparison since the NIST benchmark SV calls did not contain deletions less than $50 \mathrm{bp}$ 
Structural variation discovery in cancer genome

(Fig. S5A). We counted the overlap of these calls (Fig. S5B). As the SVs included in the NIST benchmark SV calls were counted as TP, ReALLEN and Socrates showed almost equivalent precision $(83.2 \%$ and $83.1 \%$, respectively) whereas ReALLEN called 1.3 times more SVs (762 calls) than Socrates (579 calls). The result also supports the advantage of ReALLEN.

\section{Conclusions}

In this study, we presented ReALLEN and evaluated its performance. ReALLEN showed the best score among the SR-based tools especially for detecting small SVs on low coverage data. Both RP-based methods and SR-based methods have their limitations and advantage. With long (300 bp) SE data, ReALLEN could find large translocations as effectively as the RP-based tools with short (100 bp) PE data. Moreover, the performance of ReALLEN to find small deletion and small tandem duplications was significantly superior to the RP-based tools. Consequently, ReALLEN worked very stably in most cases of our test.

ReALLEN found the CML-specific fusion gene, BCR-ABL1, on the real dataset sequenced by Ion Torrent systems. The result demonstrates the potential of ReALLEN to find such important translocations in cancer samples. Although Ion Torrent platforms are relatively low-cost, the output has a few peculiarities so that the original aligner, TMAP, is just suitable to their process. Since ReALLEN hardly depends on the first alignment, it is able to work in preferable cooperation with Ion Torrent systems. The high productivity of these platforms in combination with ReALLEN will contribute to discovery of cancer-specific fusion proteins, precise diagnosis of known types of cancers, and understanding of genetic diseases caused by abnormal chromosomes.

\section{Availability and Requirement}

Project name: ReALLEN

Project home page: https://github.com/cannaryo/reallen/

Operating system: 64-bit Linux

Programming language: Ruby 2.2.3, bash shell script

Other requirement: Ruby 2.2.3 or higher, Samtools, BWA

License: MIT

Any restrictions to use by non-academics: No

\section{List of Abbreviations}

NGS: Next Generation Sequencing; SV: Structural variation; SNV: single nucleotide variations; RP: Read-pair; RC: Read-count; SR: Split-read; AS: de novo assemble; WGS: Whole Genome 
Sequencing; TP: True positive; FP: False positive; FN: False negative; BP: breakpoint; CML: chronic myeloid leukemia.

\section{Declarations}

\section{Ethics approval and consent to participate}

The human DNA sample used in this study was collected within U.S. and International ethical guidelines. The sample was collected under Institutional Review Board (IRB) approved protocols. The detailed information was described in ILSBIO's LETTER OF CONFIRMATION.

\section{Consent for publication}

Not applicable.

\section{Availability of data and materials}

The datasets used and/or analyzed during the current study available from the corresponding author on reasonable request.

\section{Competing interests}

No competing interests.

\section{Funding}

This work was supported by the Ministry of Economy, Trade and Industry of Japan.

\section{Author's contributions}

RK designed, implemented, and tested the software and wrote the manuscript. DT and HN acquired the sequence data that helped the improvement of the software. TI supervised the project and gave suggestions about the study. All authors read and approved the final manuscript.

\section{Acknowledgements}

We thank M. Takahashi, T. Goto, M. Kawabata, and Y. Hashimoto for contribution to technical assistance. We also thank S. Watanabe, J. Imai, E. Ito, and M. Morisawa for efficient discussion.

\section{References.}

1. Stratton MR, Campbell PJ, Futreal PA. The cancer genome. Nature. 2009; 458(7239): 719-724.

\section{Tattini L, D'Aurizio R, Magi A. Detection of Genomic Structural Variants from} Next-Generation Sequencing Data. Front in Bioeng Biotechnol. 2015; 3(June): 1-8.

3. Feuk L, Carson AR, Scherer SW. Structural variation in the human genome. Nat Rev Genet. 2006; 7(February): 85-97.

4. Macconaill LE, Garraway LA. Clinical Implications of the Cancer Genome. Journal of Clinical Oncology. 2010; 28(35): 5219-28. 
5. Popovici C, Cailleres S, David M, Lafage-Pochitaloff M, Sainty D, Mozziconacci MJ. E6a2 BCR-ABL fusion with BCR exon 5-deleted transcript in a Philadelphia positive CML responsive to Imatinib. Leuk Lymphoma. 2005; 46(9):1375-7.

6. Bahassi el M, Stambrook PJ. Next-generation sequencing technologies: breaking the sound barrier of human genetics. Mutagenesis. 2014; 29(5):303-10

7. Liu L, Li Y, Li S, Hu N, He Y, Pong R, et al. Comparison of next-generation sequencing systems. J Biomed Biotechnol. 2012; doi: 10.1155/2012/251364

8. Liu B, Conroy JM, Morrison CD, Odunsi AO, Wei L, Trump DL, et al. Structural variation discovery in the cancer genome using next generation sequencing : Computational solutions and perspectives. Oncotarget. 2015; 6(8): 5477-89.

9. Rausch T, Zichner T, Schlattl A, Stütz AM, Benes V, Korbel JO. DELLY: Structural variant discovery by integrated paired-end and split-read analysis. Bioinformatics. 2012; 28(18), $333-9$.

10. Layer RM, Quinlan AR, Hall IM, Hall IM. LUMPY : A probabilistic framework for structural variant discovery. Genome Biol. 2014; doi: 10.1186/gb-2014-15-6-r84.

11. Jiang Y, Wang Y, Brudno M. PRISM: Pair-read informed split-read mapping for base-pair level detection of insertion, deletion and structural variants. Bioinformatics. 2012; 28(20): 2576-83.

12. Chen K, Wallis JW, McLellan MD, Larson DE, Kalicki JM, Pohl CS, et al. BreakDancer: an algorithm for high-resolution mapping of genomic structural variation. Nat Methods. 2009; 6(9):677-81.

13. Schröder J, Hsu A, Boyle SE, Macintyre G, Cmero M, Tothill RW, et al. Socrates: Identification of genomic rearrangements in tumour genomes by re-aligning soft clipped reads. Bioinformatics. 2014; 30(8): 1064-72.

14. Wang J, Mullighan CG, Easton J, Roberts S, Ma J, Rusch MC, et al. CREST maps somatic structural variation in cancer genomes with base-pair resolution. Nat Method. 2012; 127(8): 358-66. 
15. Zhang ZD, Du J, Lam H, Abyzov A, Urban AE, Snyder M, Gerstein M. Identification of genomic indels and structural variations using split reads. BMC Genomics. 2011; 12: $375-86$.

16. Trappe K, Emde A, Ehrlich H, Reinert K. Sequence analysis Gustaf : Detecting and correctly classifying SVs in the NGS twilight zone. Bioinformatics. 2014; 30(24): 3484-3490.

17. Karakoc E, Alkan C, O’Roak BJ, Dennis MY, Vives L, Mark K, et al. Detection of structural variants and indels within exome data. Nature Methods. 2011; 9(2): 176-8.

18. Suzuki S, Yasuda T, Shiraishi Y, Miyano S, Nagasaki M. ClipCrop : a tool for detecting structural variations with single-base resolution using soft-clipping information. $B M C$ bioinformatics. 2011; 12(Suppl 14): 1-9.

19. Ordulu Z, Wong KE, Currall BB, Ivanov AR, Pereira S, Althari S, et al. Describing sequencing results of structural chromosome rearrangements with a suggested next-generation cytogenetic nomenclature. American Journal of Human Genetics. 2014; 94(5): 695-709.

20. Li H, Durbin R. Fast and accurate long-read alignment with Burrows-Wheeler transform. Bioinformatics. 2010; 26(5): 589-95.

21. Li H. Aligning sequence reads, clone sequences and assembly contigs with BWA-MEM. 2013. arXiv:1303.3997v2.

22. Pleasance ED, Cheetham RK, Stephens PJ, McBride DJ, Humphray SJ, Greenman, CD, et al. A comprehensive catalogue of somatic mutations from a human cancer genome. Nature. 2010; 463(7278): 191-196.

23. Li H, Handsaker B, Wysoker A, Fennell T, Ruan J, Homer N, et al. The Sequence Alignment / Map format and SAMtools. Bioinformatics. 2009; 25(16), 2078-79.

24. Daley GQ, Van Etten RA, Baltimore D. Induction of chronic myelogenous leukemia in mice by the P210bcr/abl gene of the Philadelphia chromosome. Science. 1990;

247(4944):824-30. 
25. Foroni L, Gerrard G, Nna E, Khorashad JS, Stevens D, Swale B, et al. Technical aspects and clinical applications of measuring BCR-ABL1 transcripts number in chronic myeloid leukemia. Am J Hematol. 2009; 84(8): 517-22.

26. Zook JM, Catoe D, McDaniel J, Vang L, Spies N, Sidow A, et al. Extensive sequencing of seven human genomes to characterize benchmark reference materials. Sci Data. 2016; doi: 10.1038/sdata.2016.25.

27. Parikh H, Mohiyuddin M, Lam HY, Iyer H, Chen D, Pratt M, et al. svclassify: a method to establish benchmark structural variant calls. BMC Genomics. 2016; doi:

10.1186/s12864-016-2366-2.

28. 1000 Genomes Project Consortium, Abecasis GR, Auton A, Brooks LD, DePristo MA, Durbin $\mathrm{RM}$, et al. An integrated map of genetic variation from 1,092 human genomes. Nature. 2012; 491(7422): 56-65.

29. Sudmant PH, Rausch T, Gardner EJ, Handsaker RE, Abyzov A, Huddleston J, et al. An integrated map of structural variation in 2,504 human genomes. Nature. 2015; 526(7571): $75-81$.

30. Kodama Y, Shumway M, Leinonen R, International Nucleotide Sequence Database, C. The Sequence Read Archive: explosive growth of sequencing data. Nucleic Acids Res. 2012; 40: D54-56.

31. Robinson JT, Thorvaldsdottir H, Winckler W, Guttman M, Lander ES, Getz G, et al. Integrative Genomics Viewer. Nature Biotechnology. 2011; 29: 24-26.

32. Thorvaldsdottir H, Robinson JT, Mesirov JP. Integrative Genomics Viewer (IGV): high-performance genomics data visualization and exploration. Briefings in Bioinformatics. 2013; 14: 178-192

\section{Figure legends}

\section{Figure 1. ReALLEN Workflow.}

SE reads are separated to produce artificial pairs by soft-clip split or fixed-length split. Every pairs are remapped, filtered, and classified to find discordant pairs. These steps are displayed with common flowchart symbols. 
Structural variation discovery in cancer genome

Figure 2. Evaluation of ReALLEN and the other SR-based tools.

(A) F-values by Socrates, CREST, Lumpy (only SR mode), and ReALLEN for $200 \mathrm{bp} \mathrm{SE}, 300 \mathrm{bp}$ SE, and 400 bp SE on the simulated translocations (dataset \#1). The coverages are $5 \times, 10 \times, 20 \times$, and 50×. (B) F-values by Socrates, CREST, Lumpy (only SR mode), and ReALLEN for $300 \mathrm{bp}$ SE on the specific types of simulated SVs (dataset \#2). The inserted SVs are deletions, inversions, and tandem duplications ranged from 25 bp to $1600 \mathrm{bp}$. The coverage is 10×. (C) F-values by Socrates and ReALLEN for $300 \mathrm{bp} \mathrm{SE}$ on the simulated translocations (dataset \#3). Alignment \#2 (match score $=3$ ) brings longer and less precise match than Alignment \#1 (match score $=1$ ). The coverages are $5 \times, 10 \times, 20 \times$, and $50 \times$.

Figure 3. Evaluation of ReALLEN and the RP-based tools.

(A) F-values by Delly and Lumpy (SR+PE mode) for 100 bp PE and ReALLEN for 300 bp SE on the simulated translocations (dataset \#1). The coverages are $5 \times, 10 \times, 20 \times$, and $50 \times$. The insert size of $\mathrm{PE}$ is 300 bp (sigma=50). (B) F-values by Delly and Lumpy (SR+PE mode) for $100 \mathrm{bp} \mathrm{PE}$ and by ReALLEN for 300 bp SE on the specific types of simulated SVs (dataset \#2). The inserted SVs are deletions, inversions, and tandem duplications ranged from $25 \mathrm{bp}$ to $1600 \mathrm{bp}$. The coverage is $10 \times$. The insert size of PE is $300 \mathrm{bp}$ (sigma $=50$ ).

Figure 4. The translocation found in the CML sample.

(A) The junction sequence around the translocation found in the CML sample. Either ends of the sequence across the BP are matched to the region of ABL1 (ENST00000372348) and the region of BCR (ENST00000305877). (B) Schematic view of the $2.3 \mathrm{M}$ bp insertion identified with a combination of the three translocations in the CML sample. The three translocations are labeled as I, II, or III. (C) The discovery of the three SVs by ReALLEN and the other tools. Each translocation represented as I, II, or III is corresponding to the label in Figure 4B.

Figure 5. Number of SVs found in the CML sample by ReALLEN and Socrates.

(A) Venn diagram that indicates the total number of SVs detected by ReALLEN, Socrates, and their overlap in the CML sample. Two calls are considered to overlap if they have the same orientation and the locations within $20 \mathrm{bp}$. (B) Number of SVs for each type and each size category detected by ReALLEN, Socrates, and their overlap in the CML sample. The types and the size categories are the same as dataset $\# 2$. 


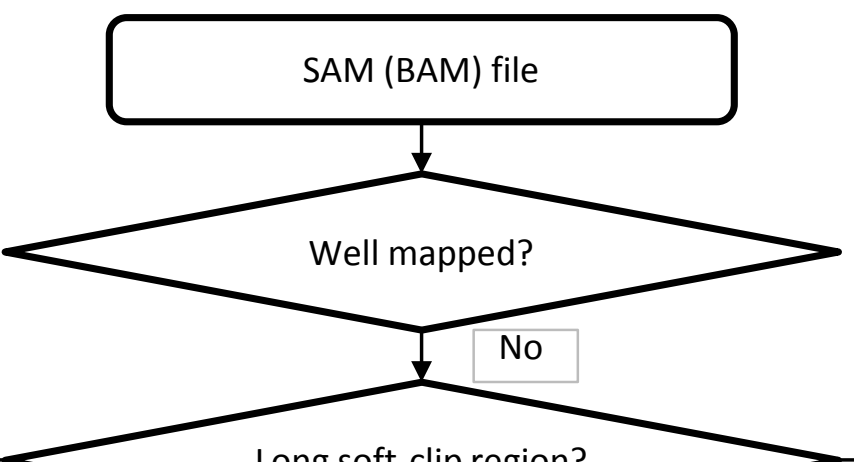

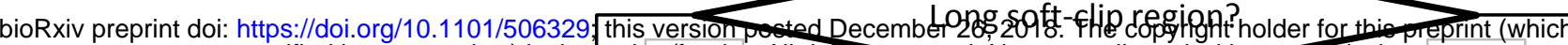

was not certified by peer review) is the author/ffynder. All rights mesed. No reuse allowed withermission. Yes

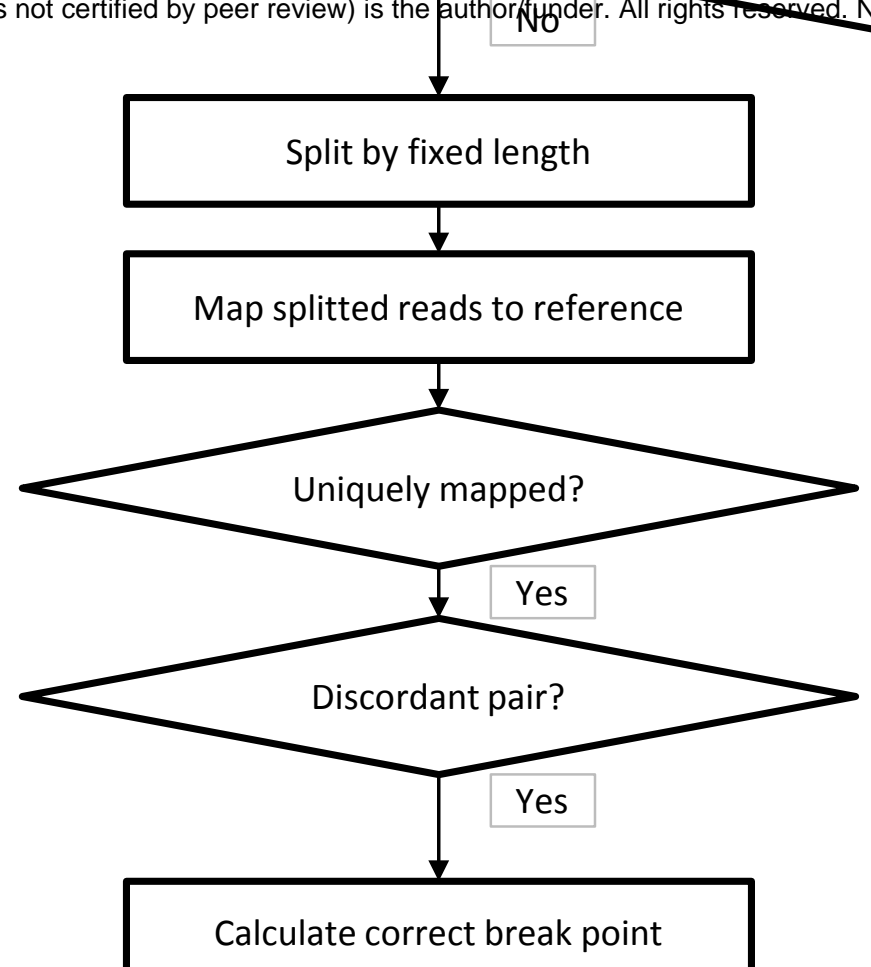

Calculate correct break point

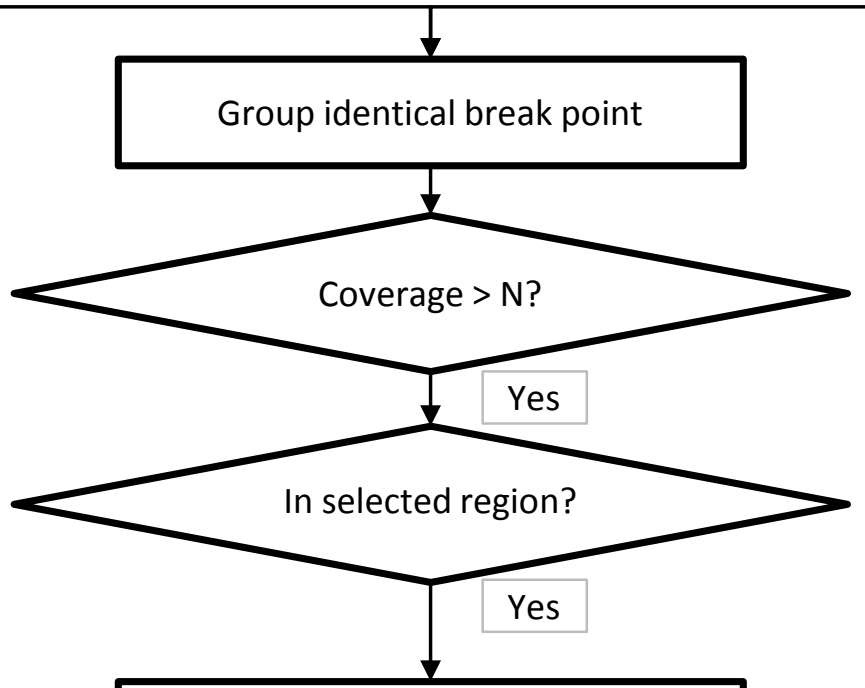

Add scores and annotations

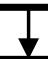

Output results to table 
A

Translocation

\section{0bp single-end 300bp single-end $\quad$ 400bp single-end}

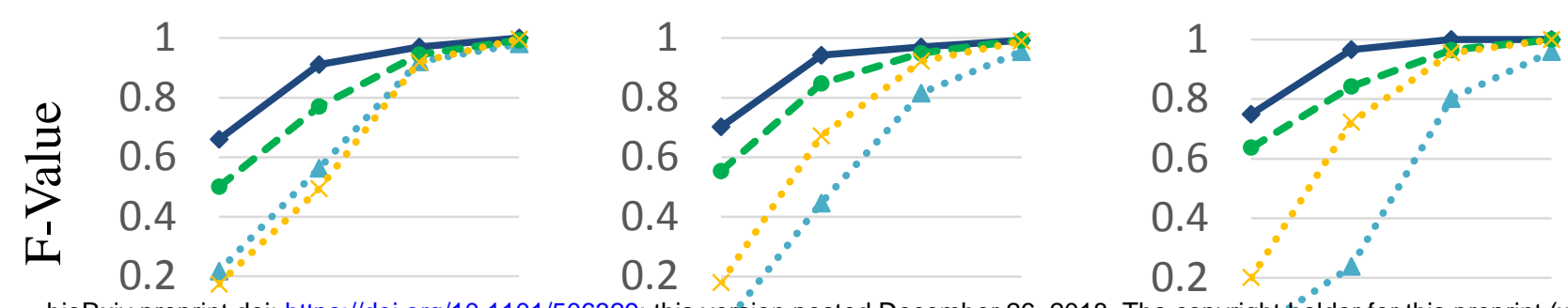

bioRxiv preprint doi: https://doi.org/10.1101/506329; this version posted December 26, 2018. The copyright tholder for this preprint (which was not certified by peer review) is the authorffunder. All rights reserved. No reuse alldured without permission.
$5 \times 10 x \quad 20 x \quad 50 x$
$5 \mathrm{x} 10 \mathrm{x} 20 \mathrm{x} 50 \mathrm{x}$
$5 x \quad 10 x \quad 20 x \quad 50 x$

\section{Mean Coverage}

B

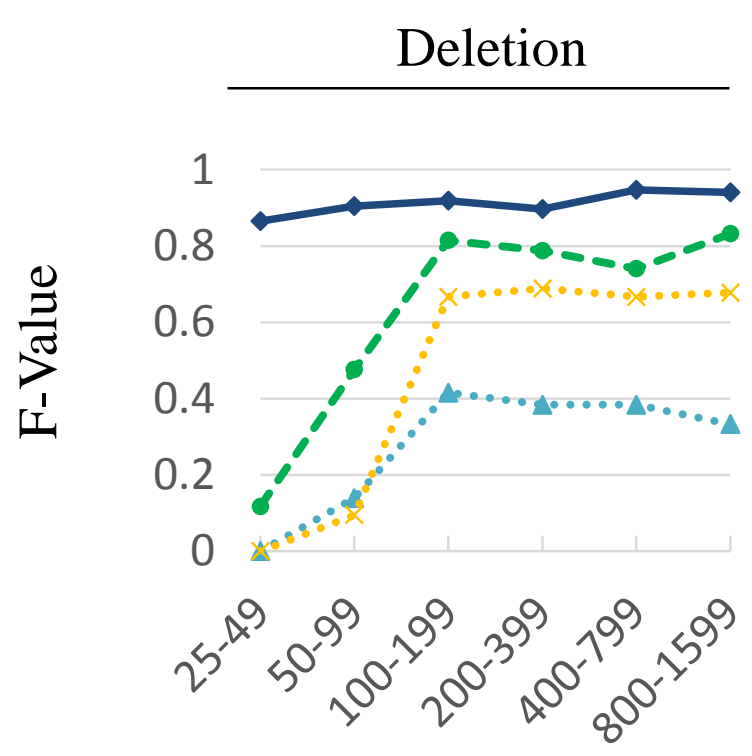

Tandem duplication

Inversion
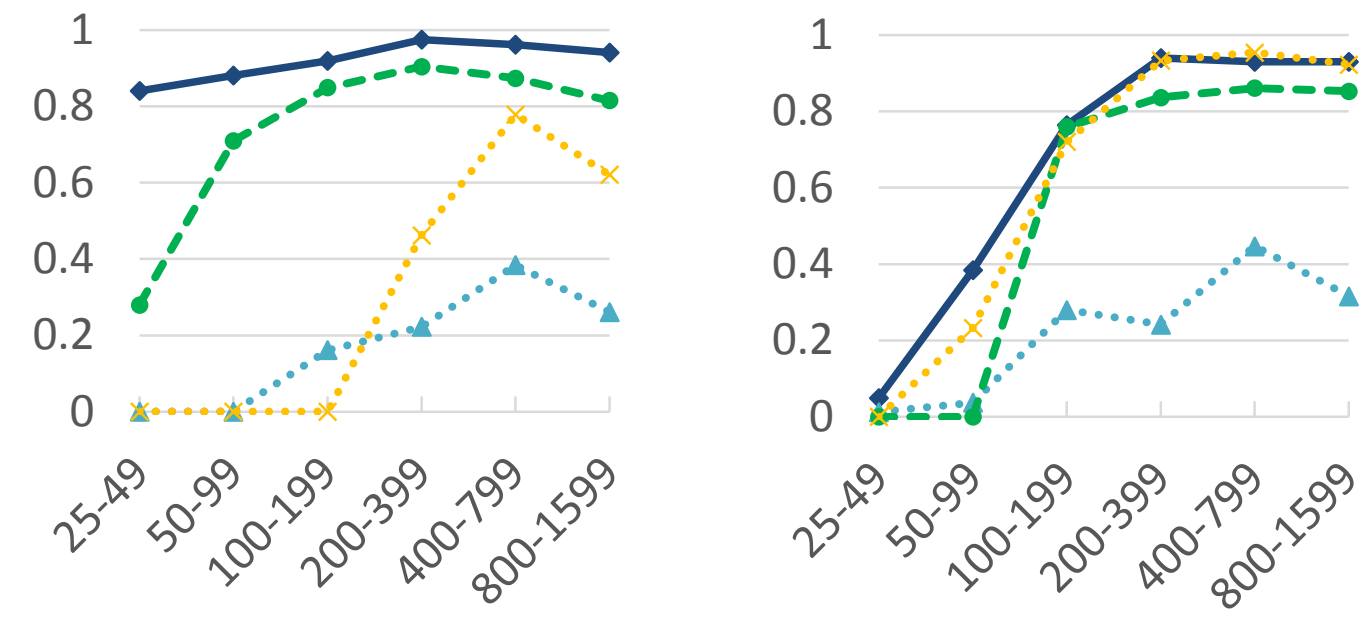

Size of structural variation [bp]

C

Translocation

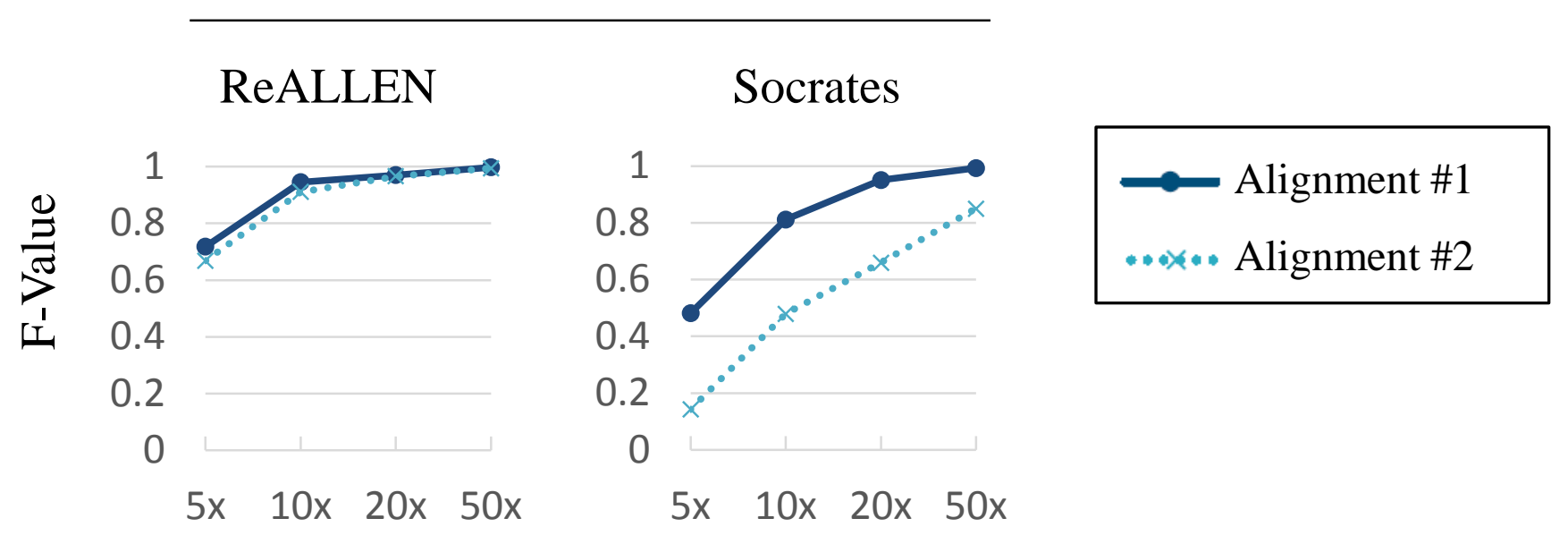

Mean Coverage 
A

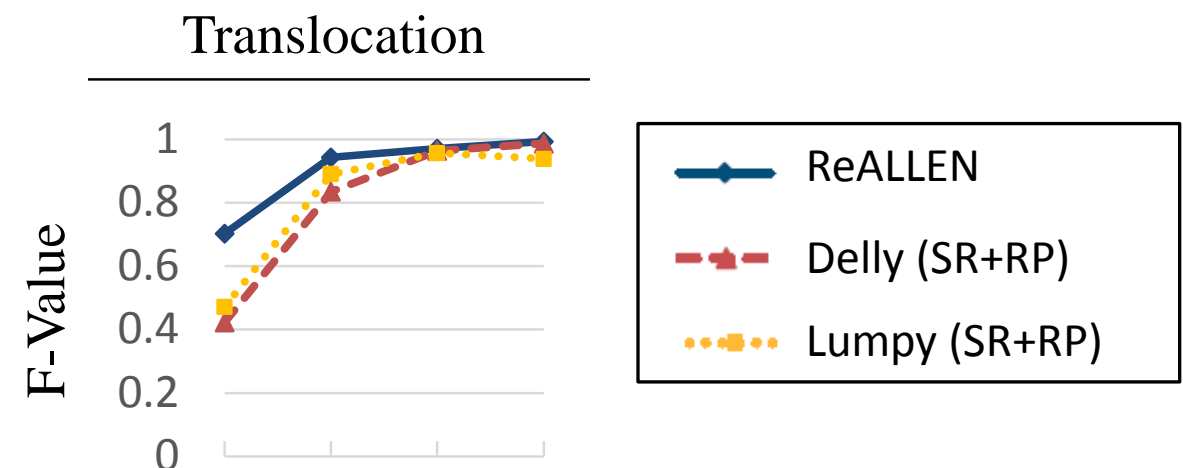

bioRxiv preprint do5 bttps:/1dgijorg/to61101/506329; this version posted December 26, 2018. The copyright holder for this preprint (which was not certified by peer review) is the author/funder. All rights reserved. No reuse allowed without permission.

\section{Mean Coverage}

B

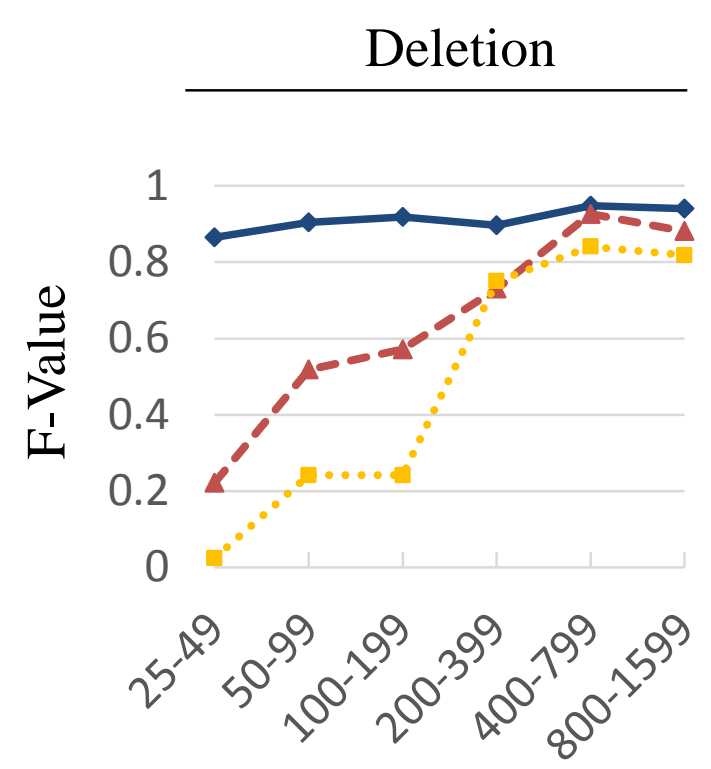

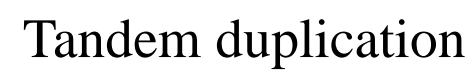
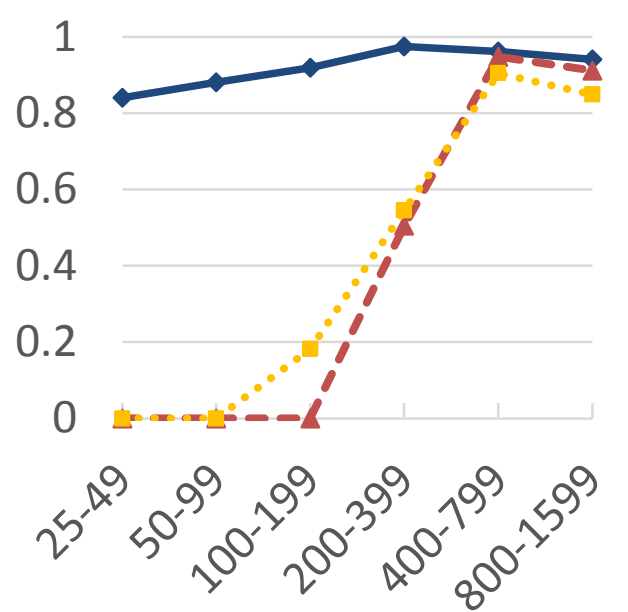

Inversion

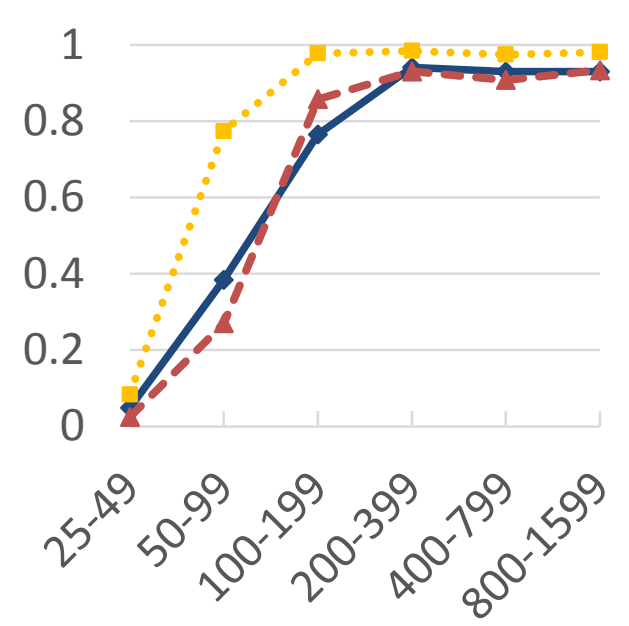

Size of structural variation [bp] 


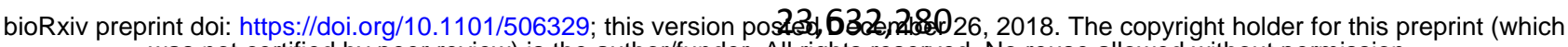
was not certified by peer review) is the author/funder. All rights reserved. No reuse allowed without permission.

B

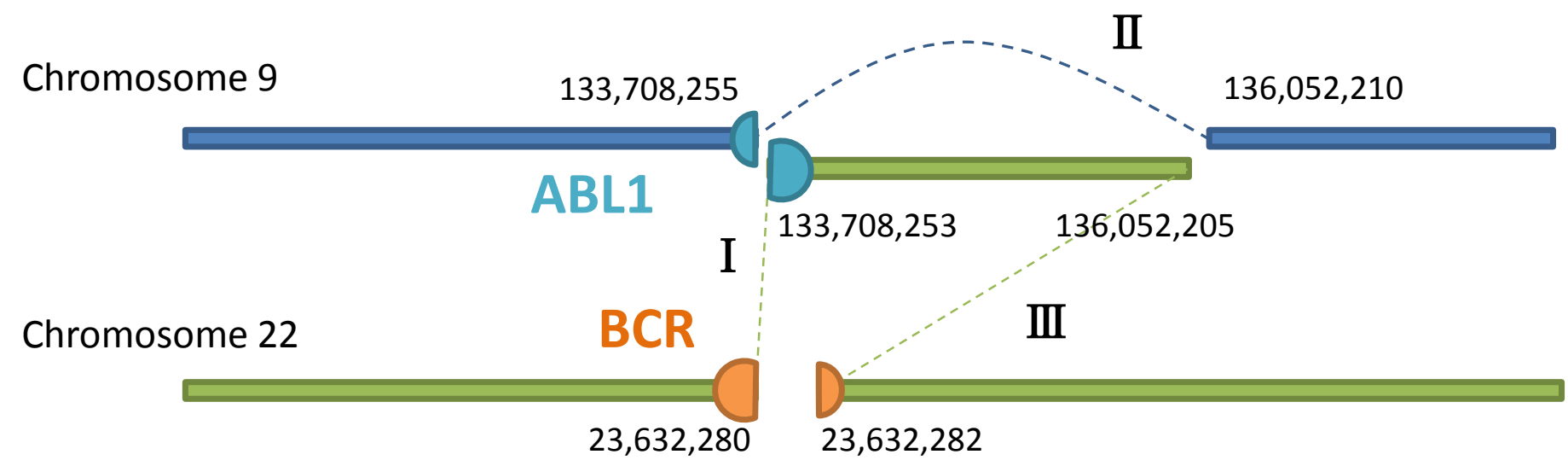

C

$\begin{array}{lccc} & \text { I } & \text { II } & \text { III } \\ \text { ReALLEN } & \checkmark & \checkmark & \checkmark \\ \text { Socrates } & \checkmark & \checkmark & \times \\ \text { CREST } & \checkmark & \times & \checkmark \\ \text { Lumpy } & \checkmark & \times & \checkmark\end{array}$


A

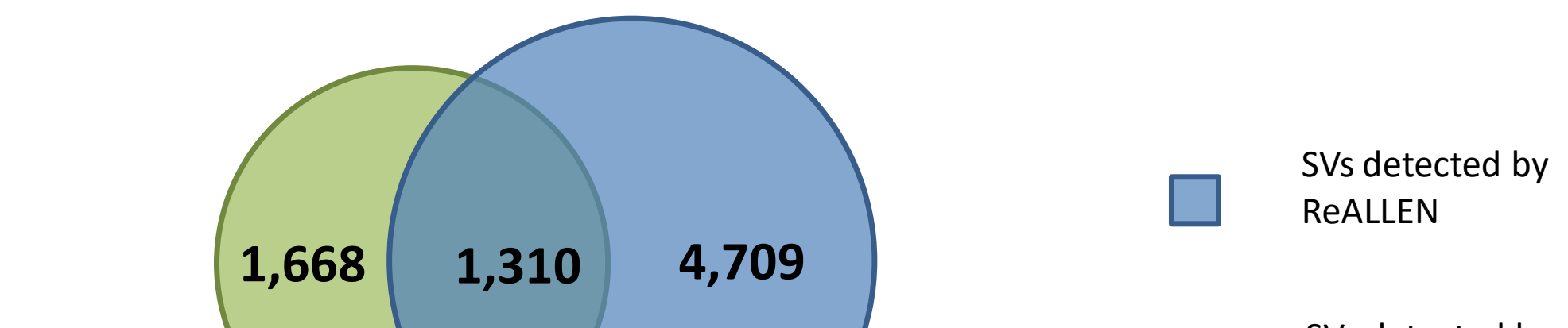

bioRxiv preprint doi: https://doi.org/10.1101/506329; this version posted December 26, 2018. The copyright holder for this preprint (whiShV detected by was not certified by peer review) is the author/funder. All rights reserved. No reuse allowed without permission.

Socrates

B

SVs detected only by ReALLEN

SVs detected only by Socrates

SVs detected by both tools

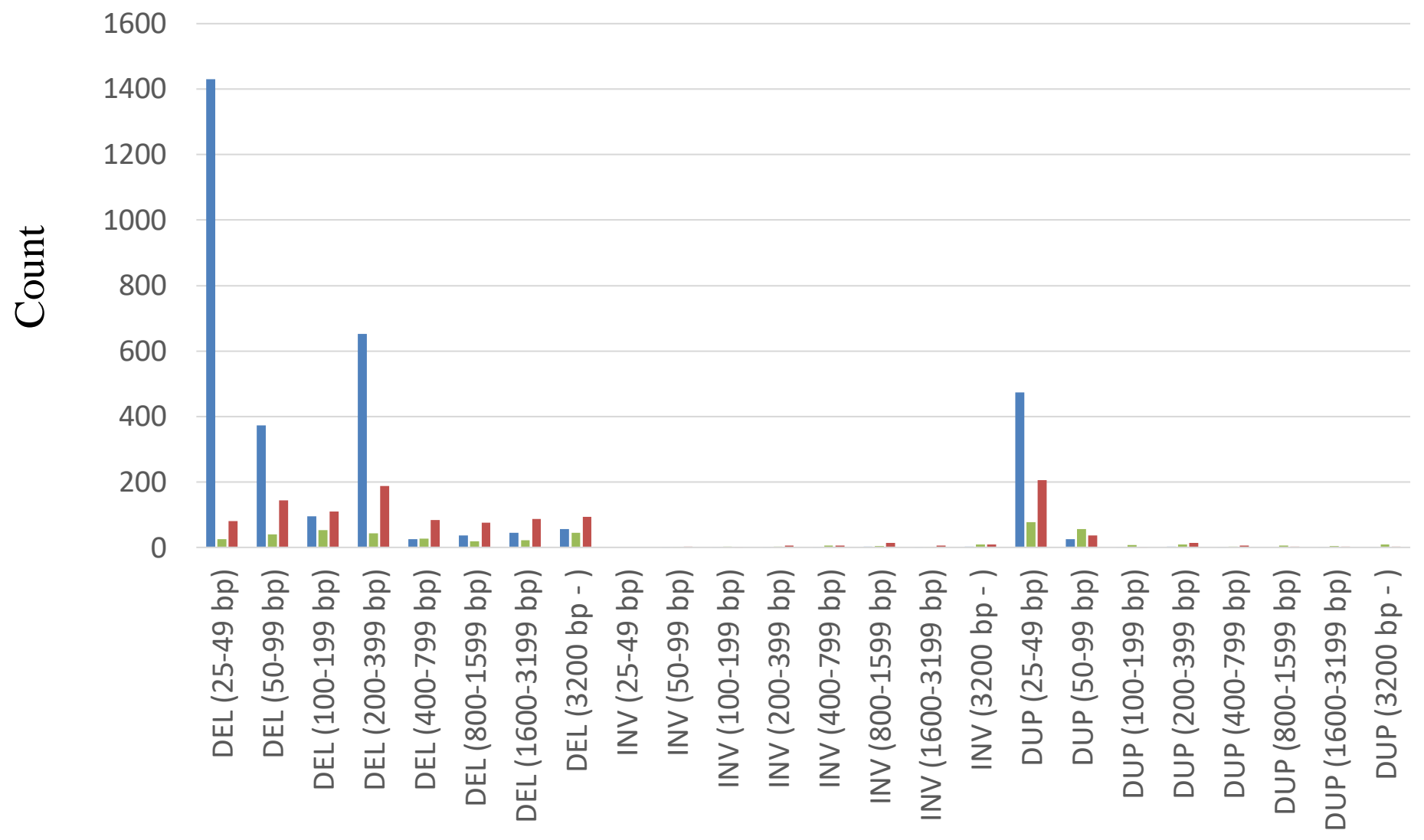

Size and type of structural variation

Figure 5. Kanno et.al. 